\title{
Direct numerical simulation of turbulent forced convection in a wavy channel at low and order one Prandtl number
}

\author{
Orsola Errico, Enrico Stalio* \\ Dipartimento di Ingegneria "Enzo Ferrari" \\ Università degli Studi di Modena e Reggio Emilia \\ Via Vignolese 905/B \\ 41125 Modena - Italy
}

\begin{abstract}
Turbulent forced convection in a channel with one planar wall and one wall of sinusoidal shape is investigated by Direct Numerical Simulation. The flow is fully developed and the Reynolds number based on the mean bulk velocity and the average hydraulic diameter is $\operatorname{Re} \approx 18900$; in this weakly turbulent flow regime three different Prandtl number values are investigated, $\operatorname{Pr}=0.025,0.20,0.71$. The fluid is in contact with the colder channel walls at an equal, uniform temperature. The main statistical quantities, like the root-mean-square of temperature fluctuations and the turbulent heat fluxes, the local heat transfer coefficient and turbulent Prandtl number values are reported. Effects of flow separation and reattachment on the local heat transfer rate and turbulent Prandtl number distribution are also presented and discussed.

An a priori analysis of the behaviour of the simple gradient diffusion model of turbulent heat fluxes is performed in the low Prandtl number, separated flow conditions of the present work. While the low Prandtl number effect can be accounted for by an appropriate selection of the turbulent Prandtl number value to be provided to the model, deviations form the expected behaviour of turbulent heat fluxes are seen to occur in the flow separation region and downstream reattachment.
\end{abstract}

\footnotetext{
*Corresponding author

Email address: enrico.stalio@unimore.it (Enrico Stalio)
} 
Key words: Turbulent convection, wavy channel, flow separation, low Prandtl number, turbulent Prandtl number 


\section{Table of contents}

\section{Contents}

1 Introduction 5

2 Governing equations $\quad 9$

2.1 Momentum equation . . . . . . . . . . . . . . . . 9 9

2.2 Energy equation . . . . . . . . . . . . . . . 10

2.3 Discrete form of the equations . . . . . . . . . . 11

3 Computational domain and mesh 11

3.1 Computational domain . . . . . . . . . . . . . . . . . 11

3.2 Computational mesh ................ 13

4 Non dimensional parameters $\quad 14$

5 Results $\quad \mathbf{1 5}$

5.1 Velocity field . . . . . . . . . . . . . 15

5.1 .1 Friction factor . . . . . . . . . . . . . 16

5.2 Temperature field . . . . . . . . . . . . . . . 16

5.2.1 Heat transfer effectiveness at different Prandtl numbers 20

5.2.2 Turbulent Prandtl number results . . . . . . . . . . . . 22

5.2.3 On the Simple Gradient Diffusion Hypothesis ... . 26

6 Conclusions 28

A Validation against experiments and previous simulations $\quad 30$ 


\section{Nomenclature}

\section{Latin letters}

\begin{tabular}{|c|c|}
\hline$a$ & wave amplitude \\
\hline$f$ & friction factor \\
\hline$f_{l}$ & friction factor of the lower wall \\
\hline$f_{u}$ & friction factor of the upper wall \\
\hline$h$ & heat transfer coefficient \\
\hline$H_{\mathrm{av}}$ & average channel height $H_{\mathrm{av}}=2 \delta$ \\
\hline$k$ & thermal conductivity \\
\hline$L_{x}, L_{z}$ & computational domain size in $x$ and $z$ directions $L_{x}=L_{z}=6 \delta$ \\
\hline$\langle\mathrm{Nu}\rangle$ & Nusselt number, calculated as in Eq. (13) \\
\hline$\langle\mathrm{Nu}\rangle_{f}$ & Nusselt Number for a flat channel \\
\hline $\begin{array}{l}\mathrm{Nu}_{l} \\
\left\langle\mathrm{Nu}_{l}\right\rangle\end{array}$ & $\begin{array}{l}\text { local Nusselt number, calculated using Eq. (14) at the lower wall } \\
\text { space averaged Nusselt number at the lower wall }\end{array}$ \\
\hline $\mathrm{Nu}_{u}$ & local Nusselt number, calculated using Eq. (14) at the upper wall \\
\hline$\left\langle\mathrm{Nu}_{u}\right\rangle$ & space averaged Nusselt number at the upper wall \\
\hline$P$ & pressure field \\
\hline$p$ & periodic part of the pressure field \\
\hline $\operatorname{Pr}$ & molecular Prandtl number, $\operatorname{Pr}=\nu / \alpha$ \\
\hline$Q_{s}$ & time-averaged volume flow rate per unit spanwise width \\
\hline $\mathrm{Pe}$ & Péclet number $\mathrm{Pe}=\mathrm{RePr}$ \\
\hline $\mathrm{Pe}^{*}$ & Péclet number of the total drag Reynolds number $\mathrm{Pe}^{*}=\mathrm{Re}^{*} \mathrm{Pr}$ \\
\hline $\mathrm{Pe}_{\tau}$ & friction Péclet number, $\operatorname{Pe}_{\tau}=\operatorname{Re}_{\tau} \operatorname{Pr}$ \\
\hline $\operatorname{Re}^{*}$ & total drag Reynolds number $\operatorname{Re}^{*}=u^{*} \delta / \nu$ \\
\hline $\operatorname{Re}_{\tau}$ & friction Reynolds number $\operatorname{Re}_{\tau}=u_{\tau} \delta / \nu$ \\
\hline $\operatorname{Re}$ & bulk Reynolds number \\
\hline$T_{\text {ref }}$ & reference temperature \\
\hline$T$ & dimensionless temperature \\
\hline$T_{b}$ & bulk temperature $T_{b}=u_{m}^{-1} \int u T \mathrm{~d} y$ \\
\hline$t$ & time \\
\hline$u^{*}$ & reference velocity $u^{*}=(\beta \delta / \rho)^{1 / 2}$ \\
\hline$u_{\tau}$ & friction velocity, $u_{\tau}=\tau_{w} / \rho$ \\
\hline$u_{m}$ & mean bulk velocity \\
\hline$u, v$ & streamwise and vertical velocity comp \\
\hline$x, y$, & vertical, and spanwise coordinate \\
\hline
\end{tabular}


Greek letters

$\alpha \quad$ thermal diffusion coefficient

$\alpha_{t} \quad$ turbulent diffusivity

$\beta \quad$ pressure drop assigned along $x$

$\delta \quad$ half the average channel height $\delta=H_{\mathrm{av}} / 2$

$\eta \quad$ wall-normal coordinate

$\lambda \quad$ wavelength

$\Lambda_{L}$ space and time-averaged temperature decay rate along the axial direction

$\nu \quad$ kinematic viscosity

$\nu_{t} \quad$ turbulent viscosity

$\rho$ density

$\tau \quad$ shear stress

$\theta \quad$ dimensionless, normalized temperature, see equation (7)

Subscripts, superscripts, symbols

- $\quad$ time average

$\langle\bullet\rangle \quad$ space average

- dimensional quantity

$\bullet_{b} \quad$ bulk quantity

- $w \quad$ wall quantity

- $w, u$ upper wall quantity

- $w, l \quad$ lower wall quantity

\section{Introduction}

Heat exchangers typically involve weakly turbulent, internal flows in corrugated, periodic geometries. The use of corrugated surfaces is motivated by the heat transfer enhancement induced by separation and reattachment phenomena. Liquid metals are characterized by very high thermal conductivity and thermal capacity. The use of liquid metals in cooling systems provides safety advantages due to their high boiling point, and the quick solidification in case of leak. Despite these premises, reliable physical models of convective heat transfer in liquid metals are still lacking, moreover the models currently employed for the more common heat transfer fluids are known to provide less accurate result for complex flows, and more specifically for flows involving separation. 
Studies dealing with the turbulent convection of heat at low Prandtl number consider in most cases the flat channel configuration. Kawamura and co-workers $[1,2]$ performed Direct numerical simulations (DNS) in the flat channel, the investigated Prandtl number values range between 0.025 and 0.71, while friction Reynolds numbers $\operatorname{Re}_{\tau}=180$ and 395 are considered. The authors observe that the effect of Re on the turbulent Prandtl number is stronger at low Pr. Instead, for $\operatorname{Pr}>0.2$ the near-wall value of turbulent Prandtl number is found to be about unity, independently of both Re and Pr. Piller et al. [3] present results of a DNS for the same range of Prandtl numbers as $[1,2]$ and for $\operatorname{Re}_{\tau}=150$. Based on temperature spectra and the correlations coefficient between velocity and temperature fluctuations, they observe that in low Prandtl number fluids the molecular conductivity acts as a filter, decreasing the effectiveness of large frequency velocity fluctuations in creating temperature fluctuations. Abe and co-workers [4] use DNS results to focus on the characteristics of surface heat flux fluctuations. Their simulations span a wide range of Re number; $\operatorname{Re}_{\tau}=180,395,640$ and 1020 and $\operatorname{Pr}=0.025$ and 0.71 . The comparison between the space-time correlations at $\operatorname{Pr}=0.71$ and 0.025 reveals that the surface heat flux fluctuations propagate downstream with a larger convection velocity for $\mathrm{Pr}=0.025$, with respect to $\operatorname{Pr}=0.71$.

A good number of studies consider the velocity field of turbulent flows over wavy surfaces, as the experimental works by Zilker and Hanratty [5], Hudson et al. [6, 7], Zenklusen et al. [8], and the numerical studies by Maaß and Schumann [9], Cherukat et al. [10], Calhoun and Street [11], Patel et al. [12]. Hudson et al. [6, 7] use a Laser Doppler Velocimetry system to investigate the fully developed turbulent flow in a rectangular water channel with a wavy bottom, having the wave amplitude to mean channel height ratio $a / H_{\mathrm{av}}=0.05$ and wavelength $\lambda=H_{\mathrm{av}}$. The Reynolds number based on half the average channel height and the bulk mean velocity is $\operatorname{Re}_{\delta}=3380$. Reversed flow is observed in the trough of the waves, both instantaneously and in the time-averaged velocity field. These works have been later used for the validation of some very detailed Direct Numerical Simulations in the same geometry. Maaß and Schumann [9] and Cherukat et al. [10] perform DNS of the turbulent flow for the same geometry and Re as in Ref. [6]. Turbulence near the wavy wall is significantly affected by the shear layer located above the recirculation region. Large velocity fluctuations are observed in the spanwise direction and velocity bursts, which originate in the separated flow regions, elongate to a large distance from the wall. Similar results have 
been reported also by von Rohr and co-workers $[13,14]$.

Experimental and numerical studies of forced turbulent convection in wavy channels are also available in the literature, but only for order one Prandtl fluids. Günther and von Rohr [15] use a Liquid Crystal Thermometry technique and, by means of Proper Orthogonal Decomposition (POD), detect the size of dominant spanwise scales of the fluid temperature. Digital Particle Image Velocimetry (PIV) and Planar Laser Induced Fluoresce (PLIF) techniques are used by Kuhn et al. [8] to study the influence of wavy walls on the passive scalar transport. They consider channels with the upper flat wall and the lower wall with waves of different shapes, for a Reynolds number of half the average channel height equal to $\operatorname{Re}_{\delta}=11200$.

Choi and Suzuki [16] performed a set of large eddy simulations of turbulent heat transfer in a channel with one wavy wall, for three different values of the wave amplitude in the range $0.01 \leq a / \lambda \leq 0.1$. The Prandtl number investigated is $\operatorname{Pr}=0.71$ and the Reynolds number is the same as in [6]. A systematic analysis of conditionally sampled turbulent thermal fields around the instantaneous streamwise vortices reveals that the primary source of vortex enhancement is the vortex stretching accompanying the near-wall flow acceleration. This greatly affects the heat transfer with the wall. The peak Nusselt number region is located at the up-slope part of the wavy wall. Instantaneous peak Nusselt events occur simultaneously with a near-wall streamwise vortex. Wagner et al. [17] performed LES of the turbulent forced convection at $\operatorname{Pr}=7$ in a channel with $2 \mathrm{D}$ or $3 \mathrm{D}$ wavy bottom wall, and for a Reynolds number of the average channel height and mean bulk velocity of 11200 and 30000 . The 2D wavy wall configuration results more effective in enhancing heat transfer than the 3D wavy wall, but the $3 \mathrm{D}$ wavy wall produces more uniform distributions of the local Nusselt number. The DNS by Rossi [18] considers the turbulent passive scalar transport in a channel with one wavy wall for the usual wave steepness of $a / \lambda=0.05$, and a Reynolds number based of half the channel average height, $R e=6850$ and Prandtl number $\operatorname{Pr}=0.71$. Dellil et al. [19] use a k- $\varepsilon$ turbulence model and a simple gradient transport model for simulating the turbulent heat transfer in the wavy walled channel. The turbulent Prandtl number value is set equal to 0.9. Waves of different amplitude are considered; it is observed that while increasing $a / \lambda$ between 0 to 0.1 , the averaged Nusselt number increases with the amplitude wave until $a \leq 0.06 \lambda$, after which it remains approximatively constant.

When computational fluid dynamics is used for practical purposes, tur- 
bulence models are employed for keeping the computational cost of simulations limited. Turbulent heat transfer models employed are usually based on the gradient diffusion hypothesis. The assessment of turbulent heat transfer models over corrugated geometries and based on the Simple Gradient Diffusion Hypothesis (SGDH), on the Generalized Gradient Diffusion Hypothesis (GGDH) model by Daly and Harlow [20] and the High Order-General Gradient Diffusion Hypothesis model by Abe and Suga [21], has already been performed by Rossi [22] for Pr $=0.71$. But turbulent convective heat transfer of fluids of low molecular Prandtl number poses the additional problem of the determination of a suitable value for $\mathrm{Pr}_{t}$, to be employed in conjunction with SGDH models. This topic has been recently investigated in few journal papers $[23,24,25,26]$ and also by Piller and coworkers [3], where the ratio of the turbulent diffusivity for generic molecular Prandtl number to the value for $\operatorname{Pr}=1$ is provided through a model. All these studies are limited to simple domains, i.e. domains not involving flow separation.

The present work aims at investigating the turbulent forced convection of low to order one Prandtl number fluids for flow configurations relevant to the typical heat exchangers passages, i.e. with a periodic set of flow separations and reattachments. The geometry selected is the same as used in many of the references mentioned above, $[6,7,9,10]$. The Reynolds number based on the mean bulk velocity and hydraulic diameter is $\mathrm{Re}=18875$ (corresponding to $\operatorname{Re}_{\delta} \approx 4720$ ); the three simulated Prandtl numbers are $\operatorname{Pr}=0.025$, representing lead-bismuth eutectic, $\operatorname{Pr}=0.2$, which corresponds to a low Prandtl number gaseous mixture, and $\operatorname{Pr}=0.71$, which corresponds to air. The main characteristics of the velocity field are shown. Results obtained for the temperature fields distribution, the temperature fluctuations, the Nusselt number and the turbulent heat fluxes are presented, also considering the Prandtl number effect. Based on the DNS data, the local turbulent Prandtl numbers are also displayed and discussed for the three temperature fields. Turbulent heat fluxes calculated by DNS are finally compared against predictions by the simple gradient diffusion model both in the cases where a single turbulent Prandtl number value, $\widehat{\operatorname{Pr}}_{t}=0.9$, is assigned for all the molecular Prandtl numbers considered, and where the turbulent Prandtl number value is assigned as function of the friction Péclet number, $\operatorname{Pr}_{t}=f\left(\mathrm{Pe}_{\tau}\right)$, as in the model proposed by Piller et al. in Ref. [3]. 


\section{Governing equations}

Governing equations are given in the following in their non-dimensional form. Dimensionless equations are obtained using half the average channel height $\delta$ as the reference quantity for length, $u^{*}=(\beta \delta / \rho)^{1 / 2}$ as the reference velocity and $t_{\text {ref }}=\delta / u^{*}$ as the reference quantity for time. In the definition of the reference velocity, $\rho$ is the density of the fluid and $\beta$ is the constant pressure drop imposed in the $x$ direction, divided by length of the computational domain in streamwise direction

$$
\frac{\langle\bar{P}\rangle_{y}(x)-\langle\bar{P}\rangle_{y}\left(x+L_{x}\right)}{L_{x}}=\beta
$$

where $\langle\bar{P}\rangle_{y}(x)$ indicates the space-averaged along $y$, time-averaged pressure field.

Temperature field is made dimensionless using the reference temperature $T_{\text {ref, }}$ while a further normalization is performed for allowing for a periodic representation of the temperature, as outlined in section 2.2.

\subsection{Momentum equation}

For the simulation of the fully developed flow in a channel, the pressure field $P$ is conveniently subdivided into a linear and an unsteady periodic contributions

$$
P(x, y, z, t)=-\beta x+p(x, y, z, t)
$$

The conservation equations for mass and momentum in dimensionless form result in

$$
\begin{gathered}
\nabla \cdot \mathbf{u}=0 \\
\frac{\partial \mathbf{u}}{\partial t}+\nabla \cdot(\mathbf{u} \otimes \mathbf{u})=-\nabla p+\frac{1}{\operatorname{Re}^{*}} \nabla^{2} \mathbf{u}+\mathbf{b}
\end{gathered}
$$

where $\mathbf{b}$ is the unit vector in $x$ direction, since in the non-dimensional form, $\beta=1$. Re $^{*}$ can be interpreted as the total drag Reynolds number, Re $^{*}=$ $u^{*} \delta / \nu$ and it differs from the friction Reynolds number which is formed using a velocity scale representing only viscous drag effects, $u_{\tau}=\sqrt{\tau_{w} / \rho}$. 


\subsection{Energy equation}

Buoyancy effects are neglected in the present study, and the temperature variable $T$ is treated as a passive scalar. Thermophysical properties are assumed to remain constant and viscous dissipation is not accounted for. The non dimensional energy equation with no heat sources, is given by

$$
\frac{\partial T}{\partial t}+\nabla \cdot(\mathbf{u} T)=\frac{1}{\mathrm{Pe}^{*}} \nabla^{2} T
$$

where a still to be defined reference temperature $T_{\text {ref }}$ is used for the non dimensional formulation and $\mathrm{Pe}^{*}=\mathrm{Re}^{*} \mathrm{Pr}$.

Uniform wall temperature conditions are set at the solid boundaries. A normalization of the temperature field is introduced for simulating the prescribed temperature conditions, by enabling a streamwise periodic variable to be calculated instead of the actual temperature field. While the more common numerical techniques make use of the bulk temperature at every step and an iterative procedure is required for this, the technique employed in this study, directly solves the transport equation of the periodic variable $\theta$

$$
\frac{\partial \theta}{\partial t}+\nabla \cdot(\mathbf{u} \theta)=\frac{1}{\mathrm{Pe}^{*}} \nabla^{2} \theta+\left(\frac{1}{\mathrm{Pe}^{*}} \Lambda_{L}^{2}+u \Lambda_{L}\right) \theta-2 \frac{1}{\mathrm{Pe}^{*}} \Lambda_{L} \frac{\partial \theta}{\partial x}
$$

with no need of multiple step procedures. The dimensionless, normalized temperature $\theta$ is defined as

$$
\theta(x, y, z, t)=\frac{T(x, y, z, t)}{\exp \left(-\Lambda_{L} x\right)}
$$

An energy balance is used to evaluate the space averaged temperature decay rate $\Lambda_{L}$ thus closing the system of equations, see Ref. [27]. The effects of axial diffusion are included in the equation for $\Lambda_{L}$ as well as in (6) and are therefore accounted for in the solution. The recovery of the actual temperature field can be finally performed through equation (7).

The temperature-like variable $\theta$ is defined apart from a multiplicative constant associated with the selection of $T_{\text {ref }}$ which has been left undetermined up to this point. This is expressed by the following equation

$$
\theta(x, y, z, t)=\frac{1}{\widetilde{T}_{\mathrm{ref}}} \frac{\widetilde{T}(x, y, z, t)}{\exp \left(-\Lambda_{L} x\right)}
$$


where $\widetilde{T}$ indicates the dimensional temperature field. In this work the three $\theta$ fields corresponding to different molecular Pr numbers have been scaled in the post processing phase, and thus also $T_{\text {ref }}$ has been selected during post processing so that the averaged heat flux at the flat wall equals unity

$$
\left.\frac{\partial \theta}{\partial y}\right|_{w, u}=-\mathrm{Pe}_{\tau}
$$

this allows for immediate comparison between temperature fields at different Pr number and also against results in the literature where heat flux is imposed at the walls. A more detailed description of the method is given in Ref. [27].

\subsection{Discrete form of the equations}

The Finite Volume code used for the simulations is the same as used in former studies of the flow and heat transfer over corrugated surfaces [28], where the transport equation for the three velocity components are solved by a second order projection-scheme. The Crank-Nicolson scheme is used for the temporal discretization of the diffusive terms of both the momentum and the energy equations, while the second-order Adams-Bashfort scheme is used for the convective terms and for the source term of the energy equation. The spatial discretization is performed by means of second order symmetric schemes. Further details can be found in Ref. [28].

\section{Computational domain and mesh}

\subsection{Computational domain}

A three dimensional computational domain is considered in this study. It is periodic in the streamwise direction; the spanwise direction is homogeneous in the sense that turbulent statistics do not change upon a shift in that direction. In order to represent the homogeneous spanwise direction, periodic boundary conditions are enforced on all variables along z. Periodic boundary conditions are enforced on all computed variables along $x$ as well.

The computational domain is depicted in figure 1, together with the coordinate system. The shape of the wavy wall $y_{w}$ in the $x-y$ plane is described by

$$
y_{w}=a\left[1+\cos \left(\frac{2 \pi x}{\lambda}\right)\right]
$$


where $a$ is the amplitude of the wave and $\lambda$ is the wavelength. In the present investigation wavelength and amplitude are set equal to $2 \delta$ and $0.1 \delta$ respectively, where $\delta$ corresponds to half the channel average height, see figure 2 . The ratio between wave amplitude and average channel height is therefore $a / H_{\mathrm{av}}=0.05$. Geometrical parameters have been selected to match the geometry investigated in the experiments in Ref. [6] and subsequent DNSs in references [9] and [10].

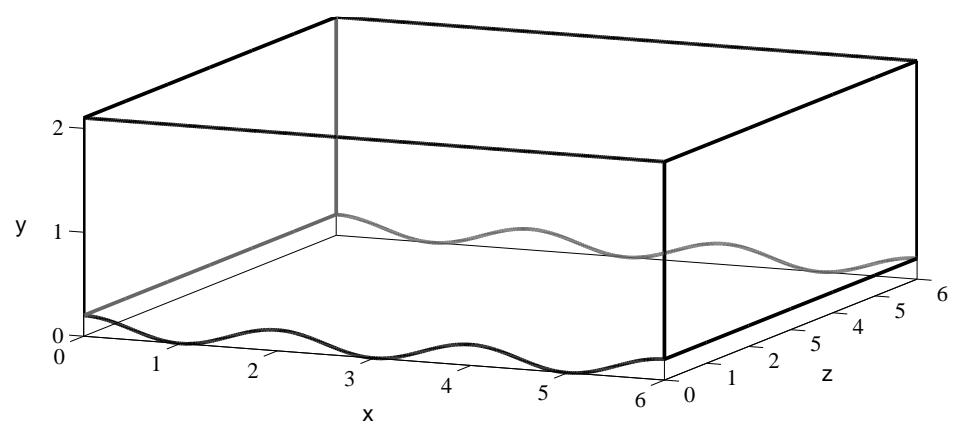

Figure 1: Periodic geometry of the problem, three dimensional view.

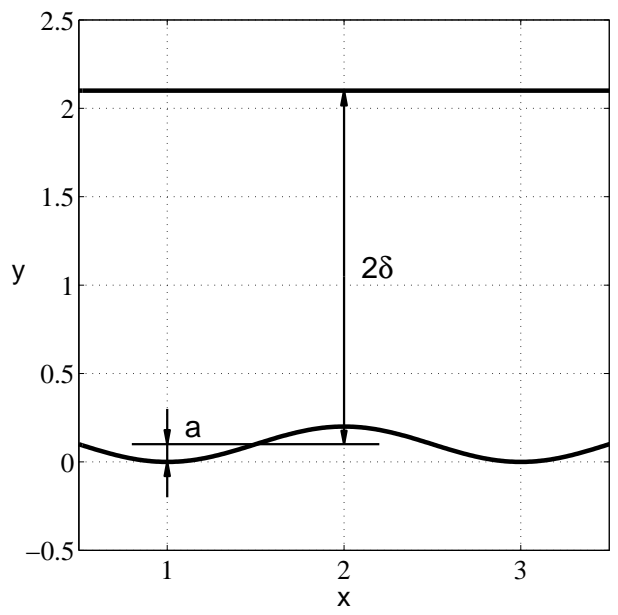

Figure 2: Two dimensional view of the computational domain. The reference length chosen is half the average channel height $\delta$.

As displayed in figure 1 the length of the computational domain in streamwise direction encompasses three periodicities, thus the domain size in $x$ is 
$L_{x}=6 \delta$. It is smaller than the domain selected in Ref. [10] where computations are performed over four periodicities, but the smaller $L_{x}$ employed does not spoil the accuracy of present results, as demonstrated by validation in A and also corroborated by the $\lambda_{2}$ analysis in Ref. [11], which shows that typical large scale structures have size in streamwise direction, $\Lambda_{x}=\lambda$. The two-point autocorrelation function in streamwise direction for the temperature field provides confirmation that the size of the computational domain in $x$ is adequate also for the $\operatorname{Pr}=0.025$ temperature field.
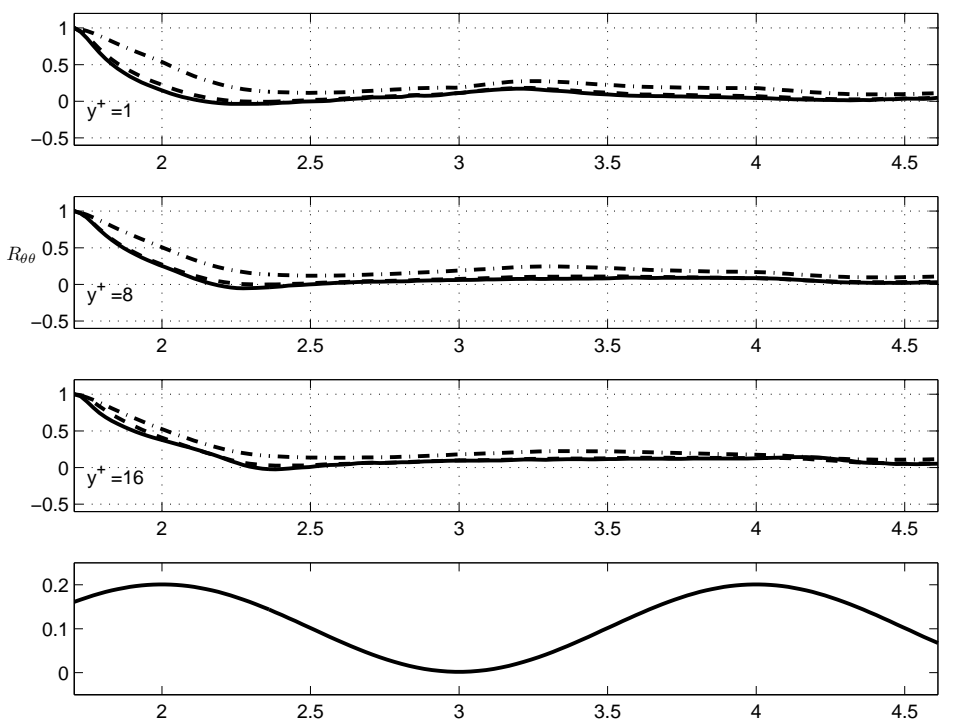

Figure 3: Two-point streamwise correlations of the temperature field at different $y^{+}$from the wavy wall. Solid line: $\operatorname{Pr}=0.71$; dashed line: $\operatorname{Pr}=0.20$; Dash-dot line: $\operatorname{Pr}=0.025$.

The width of the domain $L_{z}=3 \lambda$ is instead 1.5 times larger with respect to the investigation by Cherukat et al. [10]. This choice is based first on the spanwise two-point correlation coefficients study [4] for turbulent convection in a flat channel at $\operatorname{Pr}=0.025$, from which it is expected that temperature structures at comparable Péclet numbers can be as large as $L_{z}=1.5 \lambda$ (see figure $10 \mathrm{a}$ and $\mathrm{b}$ in Ref. [4]). In addition this is also motivated by the observation of very large spanwise structures $\left(\Lambda_{z}=1.5 \lambda\right)$ occurring over wavy walls, as described by Günther and von Rohr [30].

\subsection{Computational mesh}

A structured, curvilinear and orthogonal mesh is employed for domain discretization. The number of grid points set for the $\operatorname{Re}=18875\left(\operatorname{Re}_{\tau}=282\right)$ 
is $265 \times 187 \times 221$ along $x, y$ and $z$. To check the validity of the results obtained, a simulation for $\mathrm{Re}=13732$ has also been performed to match the flow regime of available published data for the same geometry investigated by Hudson [6] and in Ref. [10]. The mesh employed for validation has $253 \times$ $129 \times 161$ grid points along $x, y$ and $z$.

\begin{tabular}{lcccccccc} 
& & $\operatorname{Re}^{*}$ & $\operatorname{Re}_{\tau}$ & $\Delta x^{+}$ & $\Delta y_{\text {mean }}^{+}$ & $y_{w}^{+}$ & $\Delta y_{\max }^{+}$ & $\Delta z^{+}$ \\
\hline Present & $\mathrm{Re}=18875$ & 400 & 282 & 6.7 & 3.3 & 0.53 & 5.5 & 7.9 \\
Present & $\mathrm{Re}=13732$ & 305 & 216 & 5.3 & 3.6 & 0.58 & 5.7 & 8.3 \\
Ref. [9] & $\mathrm{Re}=11400$ & - & - & 10.2 & - & 1.6 & 12.4 & 10.2 \\
\hline
\end{tabular}

Table 1: Grid spacings in wall units. For the purpose of comparison values available in Ref. [9] are also indicated.

Grid spacings for the two Reynolds numbers simulated are given in Table 1, together with the friction Reynolds number value. In the same table some details of the mesh used in Ref. [9] are also reported for comparison.

\section{Non dimensional parameters}

In the numerical code employed, the pressure drop at the ends of the channel is imposed through the total drag Reynolds number Re* value. The non-dimensional global parameters, the Reynolds number and the friction factor are evaluated in terms of non-dimensional quantities by

$$
\operatorname{Re}=2 \operatorname{Re}^{*} Q_{s} ; \quad f=\frac{4 H_{\mathrm{av}}}{u_{m}^{2} \operatorname{Re}^{*}}\left\langle\left|\frac{\partial \bar{u}}{\partial \eta}\right|_{w}\right\rangle
$$

where $Q_{s}$ is the time-averaged volume flow rate per unit spanwise width of the channel, $2 H_{\mathrm{av}}$ corresponds to the average hydraulic diameter, the bulk mean velocity is $u_{m} \equiv Q_{s} / H_{\mathrm{av}}$ and the angular brackets indicate a spatial average.

The friction Reynolds number is calculated as

$$
\operatorname{Re}_{\tau}=\frac{u_{\tau} \delta}{\nu} ; \quad u_{\tau}=\sqrt{\frac{\tau_{w, u}+\tau_{w, l}}{2 \rho}}
$$

where the wall shear stress of the upper and lower wall $\tau_{w, u}$ and $\tau_{w, l}$ are calculated on the projected horizontal area. 
The Nusselt number in terms of non dimensional quantities is evaluated from

$$
\langle\mathrm{Nu}\rangle=\frac{2 H_{\mathrm{av}}}{\left\langle T_{b}\right\rangle-T_{w}}\left\langle\left|\frac{\partial \bar{T}}{\partial \eta}\right|_{w}\right\rangle
$$

where $\eta$ is the wall normal coordinate. A local Nusselt number can be defined starting from equation (13)

$$
\mathrm{Nu}(x)=\frac{2 H_{\mathrm{av}}}{T_{b}-T_{w}}\left|\frac{\partial \bar{T}}{\partial \eta}\right|_{w}
$$

the local $\mathrm{Nu}(x)$ allows for the discussion of heat transfer performance in specific portions of the channel.

\section{Results}

After a statistically steady state is reached, fluid flow and thermal field realizations are saved every 0.05 non-dimensional time units and for a period of 12.5 flow-through times in order to form statistics. Besides being integrated in time, mean quantities presented in this section are also averaged over the waves and the spanwise length $L_{z}$.

\subsection{Velocity field}

The turbulent velocity field of the case presented here has already been investigated by different authors $[6,7,9$, 10] for a slightly lower Reynolds number. Turbulent flow features observed by previous investigators are close to those observed at $\mathrm{Re} \approx 18900$ in the present work, therefore the discussion for the velocity field is kept succinct.

Streamlines and profiles of the mean velocity field on the periodic module of the channel are depicted in figure 4, together with the indication of the mean separation and reattachment points.

A shear layer region is located above the mean recirculation region and is indicated in figure 5 by a gray area, which is the area of maximum Reynolds stress, where $-\overline{u^{\prime} v^{\prime}}$ exceed the threshold of 2.1. Reynolds stress on the upper wall are essentially independent of the streamwise coordinate. On the upslope portion of the wave, Reynolds stress become negative in a thin layer close to the wall only when calculated in the Cartesian coordinate system. As already established in previous works, see for example Ref. [7], the Reynolds stress are instead always positive in a reference frame aligned with the wavy walls. 


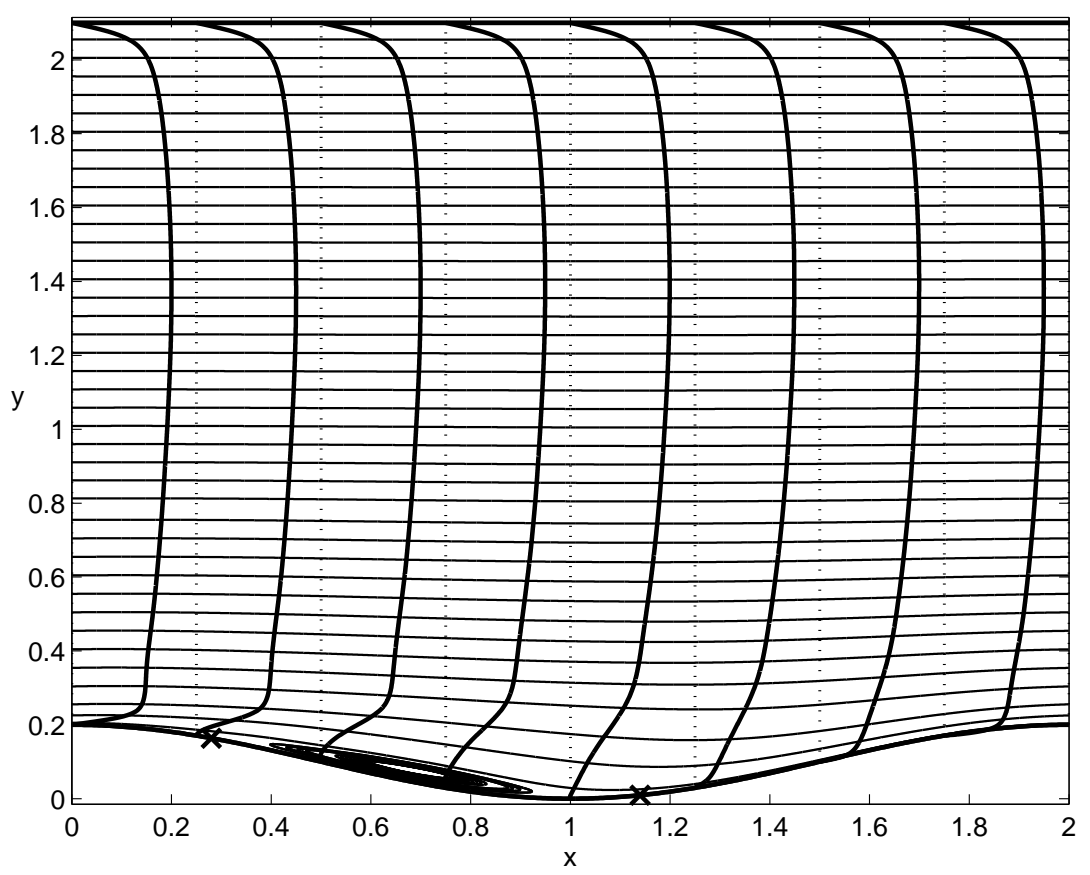

Figure 4: Profiles of the streamwise component of the mean velocity field and streamlines. Mean flow direction is from left to right. Mean separation and reattachment locations are indicated by x-symbols.

\subsubsection{Friction factor}

Friction factor values calculated as in equation (11) are reported in Table 2 together with reference values for the flat channel from the literature. Due to the presence of the separation bubble, viscous drag on the wavy wall is smaller than that calculated on the upper flat wall. Friction on the upper wall is instead larger than the value given for a flat channel case at the same Re and calculated by the following empirical relation devised by Dean [32]

$$
f=0.292 \mathrm{Re}^{-1 / 4}
$$

The closest available published data from flat channel DNSs are also reported in Table 2 for assessing the accuracy of the empirical relation used. The local friction factor at the two channel walls is reported in figure 6 .

\subsection{Temperature field}

Mean temperature profiles are shown in figure 7. Temperature profiles change their characteristics depending on the Prandtl number: while for 


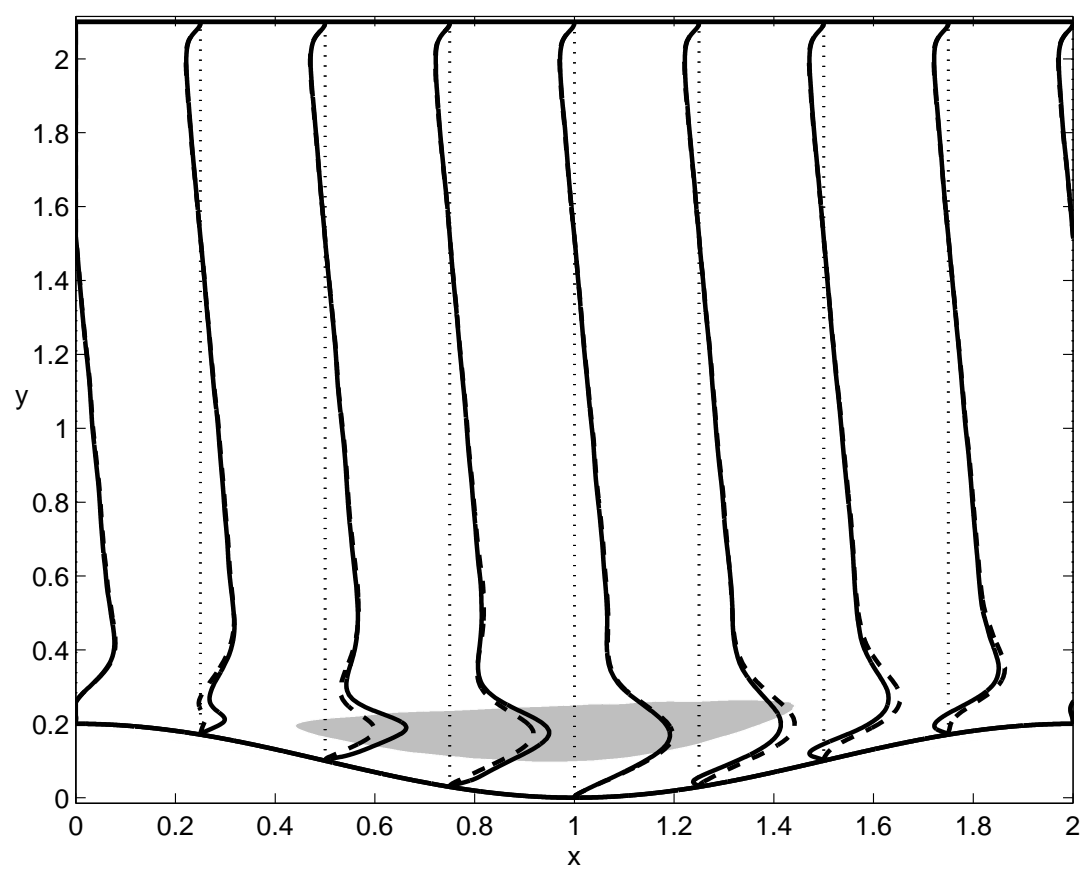

Figure 5: Profiles of the Reynolds stress $-\overline{u^{\prime} v^{\prime}}$. The gray area is the region where Reynolds stress $-\overline{u^{\prime} v^{\prime}}$ are greater than 2.1, while in the whole field $-0.50<-\overline{u^{\prime} v^{\prime}}<3.13$. The dashed lines indicate Reynolds Stress profiles calculated on a local reference frame aligned with wavy walls.

$\operatorname{Pr}=0.71$, the shape of the profile is turbulent, for $\operatorname{Pr}=0.025$ the profile shape is typically laminar. As a consequence, it is not possible to extend results presented for the low-Prandtl number cases to turbulent convection at low Prandtl number and high Péclet numbers, where not only the fluid flow is turbulent but also the temperature field displays typical turbulent features. For all Prandtl number values investigated the temperature field close to the upper wall is almost independent of the streamwise coordinate, instead the temperature field is observed to be influenced by the lower wall geometry, especially for $\operatorname{Pr}=0.71$ and $\operatorname{Pr}=0.20$. An inflection of the temperature profile is observed just above the separated flow region. It corresponds to a local minimum of the mean temperature gradient along $y$ and is to be ascribed to the cold stream originating from the crest of the wave which locally inhibits the temperature increase along $y$. This feature, which is mainly due to advective transport, is not observed for $\operatorname{Pr}=0.025$. Profiles in figure 7 are all scaled to the same peak temperature for easier comparison 


\begin{tabular}{ccccc} 
& $\begin{array}{c}\text { flat channel } \\
\text { Eq. }(15)\end{array}$ & $\begin{array}{c}\text { flat channel } \\
\text { DNS data [31] }\end{array}$ & $\begin{array}{c}\text { wavy channel } \\
\text { flat wall }\end{array}$ & $\begin{array}{c}\text { wavy channel } \\
\text { wavy wall }\end{array}$ \\
\hline $\mathrm{Re}=11172$ & 0.0284 & 0.0325 & - & - \\
$\mathrm{Re}=18875$ & 0.0249 & - & 0.0345 & 0.0247 \\
$\mathrm{Re}=28405$ & 0.0225 & 0.0244 & - & - \\
\hline
\end{tabular}

Table 2: Friction factor values in the wavy channel for the flat wall and the wavy wall. Flat channel results are reported for comparison; DNSs data at $\mathrm{Re}=11172\left(\operatorname{Re}_{\tau}=180\right)$ and $\operatorname{Re}=28405\left(\operatorname{Re}_{\tau}=395\right)$ are taken from Ref. [31]; the empirical relation by Dean is given in Ref. [32].

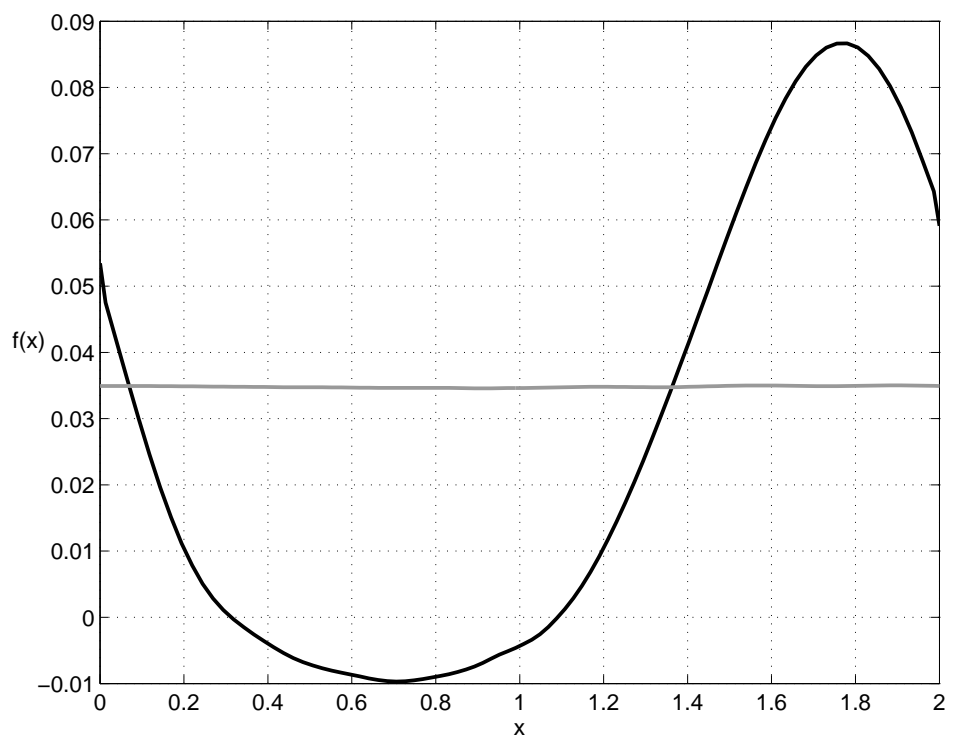

Figure 6: Profiles of the local friction factor: the black curve represents $\mathrm{f}_{l}$, while the gray line represents $\mathrm{f}_{u}$.

between profile shapes. Temperature profiles using reference quantities as indicated in equations (8) and (9) are drawn in figure 8.

Three snapshots of the temperature field, each for a different Prandtl number, are displayed in figure 9 together with the fluctuation field; all thermal fields correspond to the same velocity field, i.e. snapshots are taken at the same time-step. The size of the smallest length scale decreases significantly from $\operatorname{Pr}=0.025$ to $\mathrm{Pr}=0.71$. It is observed that the mean temperature profiles of laminar characteristics at $\operatorname{Pr}=0.025$ shown in figure 


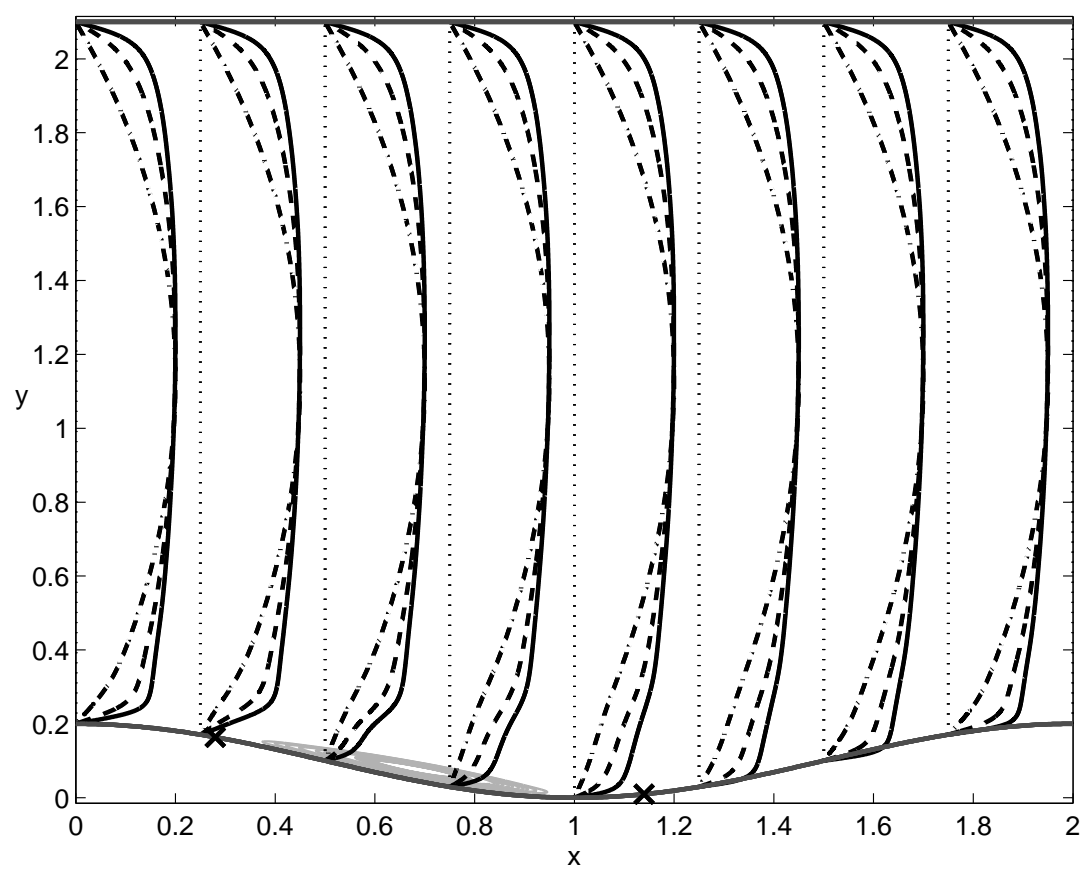

Figure 7: Profiles of the normalized mean temperature field $\theta$ for three different Prandtl numbers, solid line: $\operatorname{Pr}=0.71$; dashed line: $\operatorname{Pr}=0.20$ and dash-dot line $\operatorname{Pr}=0.025$. Mean separation and reattachment locations are indicated by $\mathrm{x}$-symbols. Streamlines of the mean vortex are also indicated in the figure.

7 correspond to an unsteady temperature field, with very little separation between the small and the large spatial scales and hence with small turbulent heat fluxes.

Profiles of the root-mean-square of the temperature fluctuations are depicted in figure 10; it is noticed that the lower wall geometry extends its effects well above the mean channel height. Close to the lower wall and for $\operatorname{Pr}=0.71$ two peaks appear at $x=0.7$, the lower peak corresponds to the region of maximum velocity of the mean recirculating bubble, while the other one corresponds to the shear layer. Profiles in the boundary layer restart region (see profiles for $x=1.3$ and $x=1.7$ in figure 10) display a sharp peak close to the lower wall, while the peak originating from the shear layer is considerably reduced. For $\operatorname{Pr}=0.025$ it is impossible to discriminate the two peaks as the conduction-dominated temperature field at this low Péclet number is only weakly influenced by the turbulent flow. Shapes of profiles obtained for $\operatorname{Pr}=0.20$ lie between the two cases discussed. 

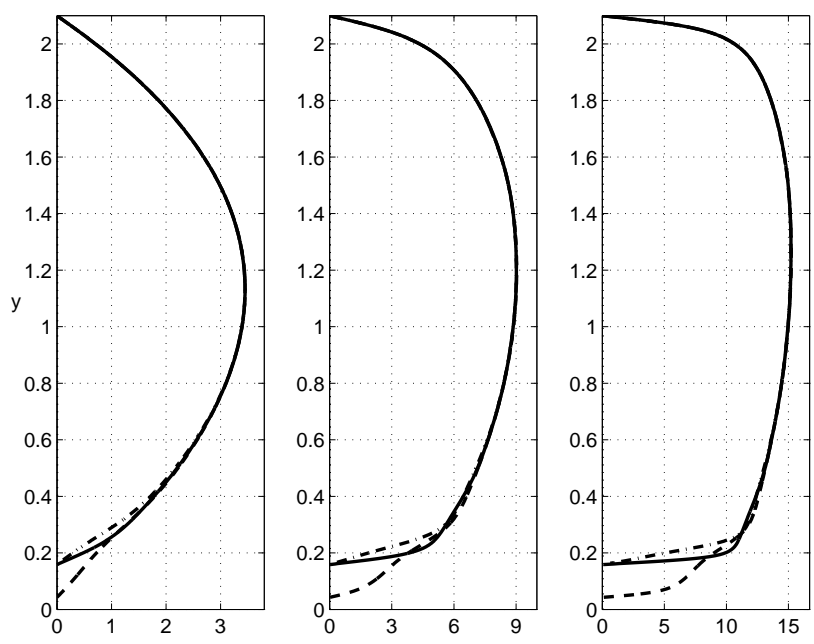

Figure 8: Profiles of the mean temperature field $\theta$ for $\operatorname{Pr}=0.025, \operatorname{Pr}=0.20$ and $\operatorname{Pr}=0.71$ in three different $x$ locations. Solid lines indicate $x=0.3$, dashed lines are for $x=0.7$ and dash-dot lines for $x=1.7$.

Turbulent heat fluxes are depicted in $x, y$ and wall normal direction in figure 11. Distribution of the turbulent heat fluxes in $x$, figure 11a, reveals that the streamwise component of turbulent heat fluxes reaches its maximum along the shear layer region. Also profiles of vertical heat fluxes, figure 11b, 11c and 11d, display their maxima along the shear layer. Like for the Reynolds stress, $\overline{v^{\prime} \theta^{\prime}}$ become locally positive on the up-slope portion of the wave only when calculated in the Cartesian reference frame. For decreasing Prandtl numbers, turbulent heat fluxes almost vanish, see figure 11d, this is in direct relation with the laminar shape of mean temperature profiles for $\mathrm{Pe}=472$.

\subsubsection{Heat transfer effectiveness at different Prandtl numbers}

Local Nusselt numbers, calculated as in equation (14), are displayed in figure 12 for the three fluids considered and for the two channel walls. Peak heat transfer rates are always located in the up-slope portion of the lower wall, past the flow reattachment location; the insulating effect of vortices has already been discussed in [29]. Deviations from the space-averaged values due to flow characteristics like separation, reattachment and shear layer formation decrease for decreasing Pr.

Table 3 reports the global Nusselt numbers calculated from the present DNS together with the $\mathrm{Nu}$ values for the flat channel $\langle\mathrm{Nu}\rangle_{f}$, calculated using 


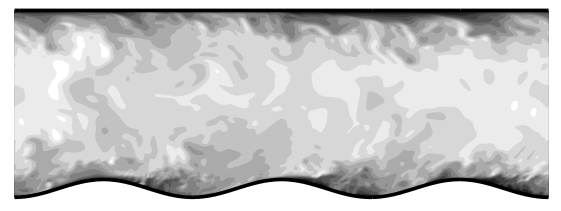

(a) $\theta$ at $\operatorname{Pr}=0.71$

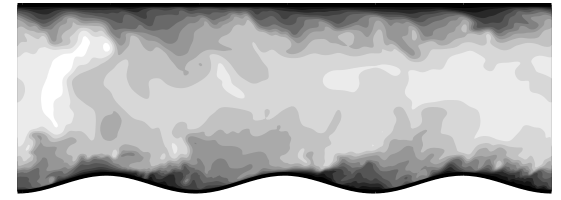

(c) $\theta$ at $\operatorname{Pr}=0.20$

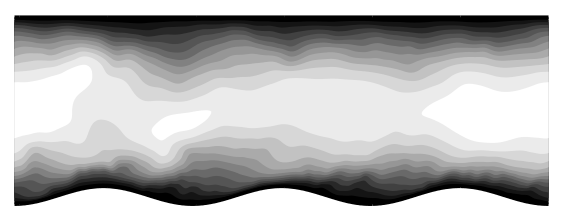

(e) $\theta$ at $\operatorname{Pr}=0.025$

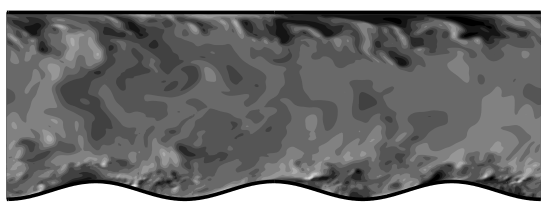

(b) $\theta^{\prime}$ at $\operatorname{Pr}=0.71$

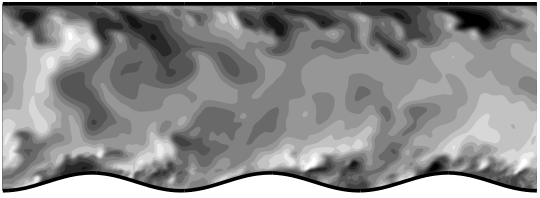

(d) $\theta^{\prime}$ at $\operatorname{Pr}=0.20$

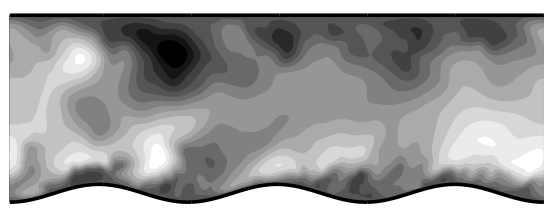

(f) $\theta^{\prime}$ at $\operatorname{Pr}=0.025$

Figure 9: Snapshots of the instantaneous temperature field: $\theta$ in a,c,e; $\theta^{\prime}$ in b,d,f. Temperature fluctuations at Prandtl 0.71 displayed in inset b range between $-0.35 \leq \theta^{\prime} \leq 0.77$; temperature fluctuations at Prandtl 0.20 displayed in inset d range between $-0.33 \leq$ $\theta^{\prime} \leq 0.55$; temperature fluctuations at Prandtl 0.025 displayed in inset $\mathrm{f}$ range between $-0.25 \leq \theta^{\prime} \leq 0.51$.

the correlation by Sleicher and Rouse [33] and the heat transfer ratio $h_{r}$, defined as $h_{r} \equiv\langle\mathrm{Nu}\rangle /\langle\mathrm{Nu}\rangle_{f}$. The $x$-averaged Nusselt number at the upper wall and lower wall $\langle\mathrm{Nu}\rangle_{u}$ and $\langle\mathrm{Nu}\rangle_{l}$ are as well reported in the table. Notice that although introduced for pipe flow, the correlation in Ref. [33] can also be applied to the flat channel case [1].

As already established for the flat channel geometry [1], heat transfer effectiveness of low Prandtl number fluids is low. Heat transfer enhancement do to the presence of the wavy wall is instead substantial. It is apparent from table 4 that the heat transfer ratio is largest for $\operatorname{Pr}=0.20$ and smaller for $\operatorname{Pr}=0.71$ and $\operatorname{Pr}=0.025$. The heat transfer enhancement obtained is compensated by a corresponding increase in friction drag, see table 2 , and in the addition of a form drag whose measure is in the difference between the total drag velocity $u^{*}$ and the friction velocity $u_{\tau}$. 

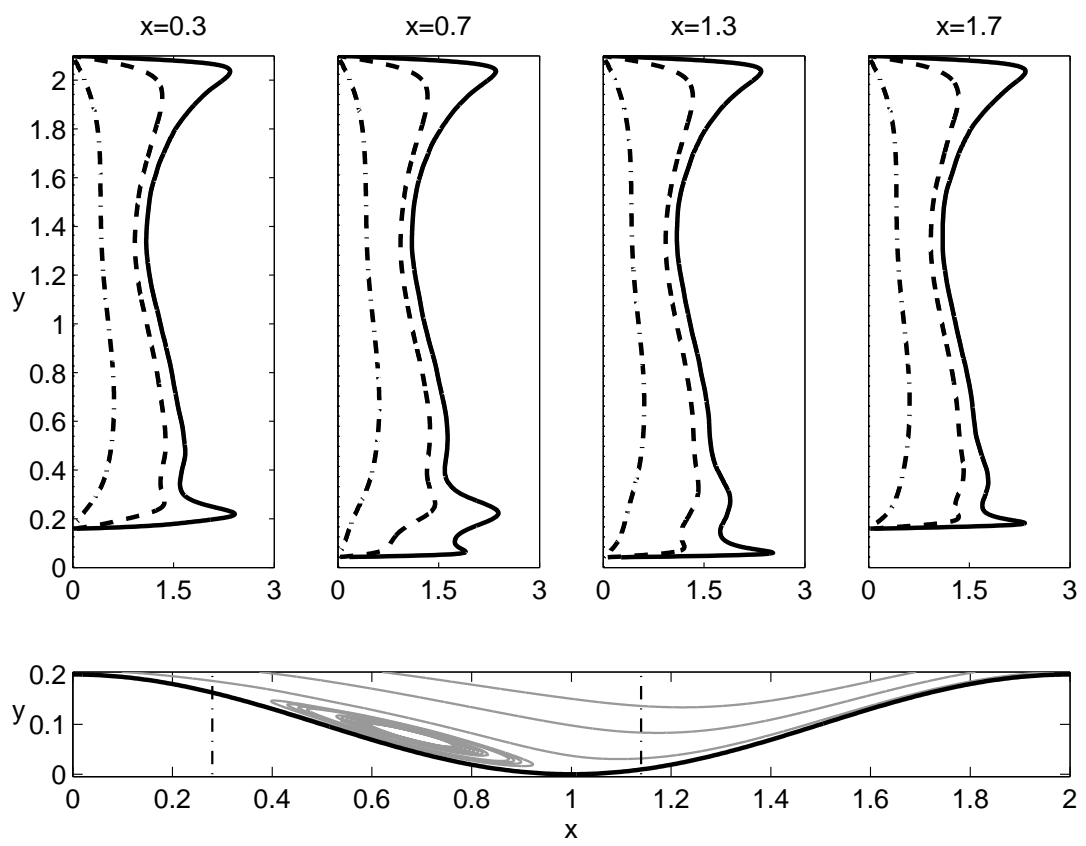

Figure 10: Root-mean-square of the temperature fluctuations, $\theta_{\mathrm{rms}}$, solid line: $\operatorname{Pr}=0.71$; dashed line: $\operatorname{Pr}=0.20$ and dash-dot line $\operatorname{Pr}=0.025$.

\begin{tabular}{lcccccc}
$\operatorname{Pr}$ & $\Lambda_{L}$ & $\begin{array}{c}\langle\mathrm{Nu}\rangle \\
\text { Eq. (13) }\end{array}$ & $\begin{array}{c}\langle\mathrm{Nu}\rangle_{f} \\
\text { Ref. [33] }\end{array}$ & $\langle\mathrm{Nu}\rangle /\langle\mathrm{Nu}\rangle_{f}$ & $\left\langle\mathrm{Nu}_{l}\right\rangle$ & $\left\langle\mathrm{Nu}_{u}\right\rangle$ \\
\hline 0.71 & 0.0058 & 77.3 & 47 & 1.64 & 99.1 & 63.0 \\
0.20 & 0.0099 & 37.1 & 19 & 1.95 & 48.9 & 33.5 \\
0.025 & 0.026 & 12.2 & 7.3 & 1.67 & 15.9 & 13.6 \\
\hline
\end{tabular}

Table 3: Collection of heat transfer results for $\mathrm{Re}=18875$ and different Prandtl numbers. The table provides temperature decay rate, Nusselt number of the wavy channel, Nusselt number for the flat channel after Ref. [33], heat transfer ratio, $x$-averaged value of $\mathrm{Nu}_{u}$ and $x$-averaged value of $\mathrm{Nu}_{l}$.

\subsubsection{Turbulent Prandtl number results}

The turbulent Prandtl number is a fundamental parameter for practical heat transfer analyses, it is defined as follows

$$
\operatorname{Pr}_{t} \equiv \frac{\overline{u^{\prime} v^{\prime}} \frac{\partial \bar{T}}{\partial y}}{\overline{v^{\prime} T^{\prime}} \frac{\partial \bar{u}}{\partial y}}
$$



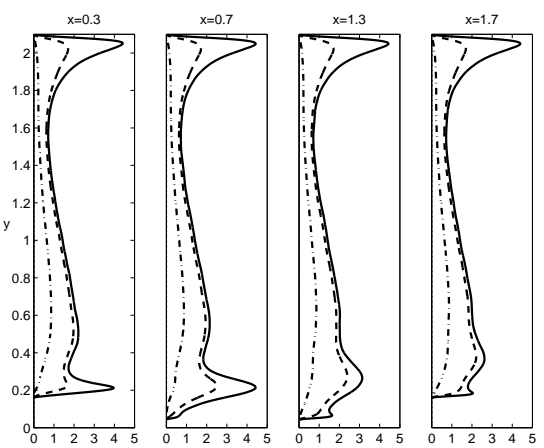

(a) $\overline{u^{\prime} \theta^{\prime}}$ at $\operatorname{Pr}=0.025,0.20,0.71$

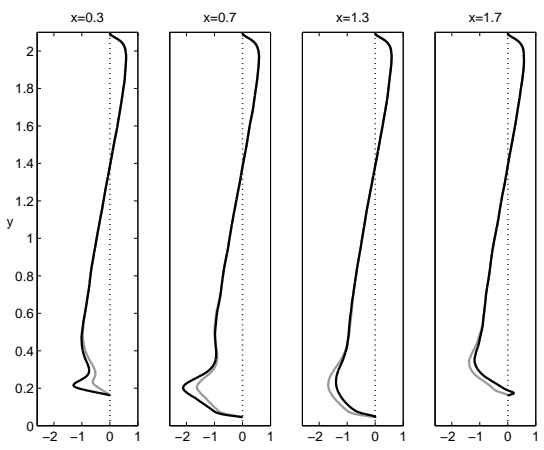

(b) $\overline{v^{\prime} \theta^{\prime}}$ at $\operatorname{Pr}=0.71$

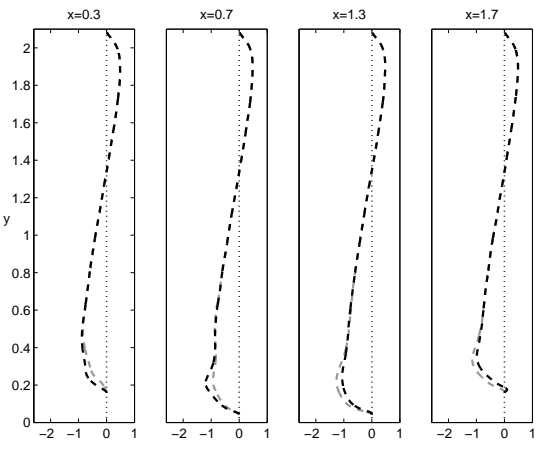

(c) $\overline{v^{\prime} \theta^{\prime}}$ at $\operatorname{Pr}=0.20$

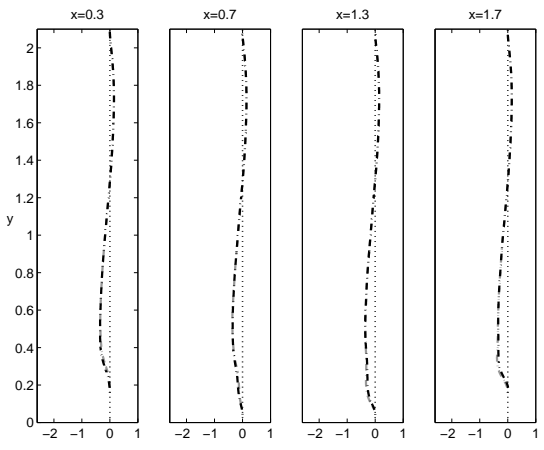

(d) $\overline{v^{\prime} \theta^{\prime}}$ at $\operatorname{Pr}=0.025$

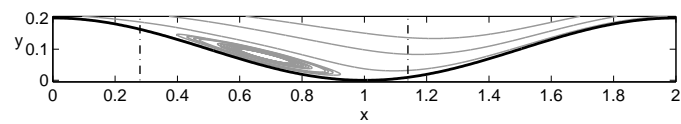

Figure 11: Profiles of turbulent heat fluxes, (a) $\overline{u^{\prime} \theta^{\prime}}$ : solid line $\operatorname{Pr}=0.71$; dashed line $\operatorname{Pr}=0.20$; dash-dot line $\operatorname{Pr}=0.025$. Insets (b) (c) (d), black lines: vertical heat fluxes; gray lines: heat fluxes calculated on a reference frame locally aligned with the wavy walls.

Contour plots of the turbulent Prandtl number calculated from equation (16) are depicted in figure 13 .

Gray levels are reported between $0.50<\operatorname{Pr}_{t}<3.3$, white regions instead indicate that $\operatorname{Pr}_{t}$ value is beyond those limits. The limits have been selected on the basis of Fig. 9, Ref. [1], where $\operatorname{Pr}_{t}$ profiles are displayed for the flat channel. This allows to emphasize regions where the local $\mathrm{Pr}_{t}$ exceeds the range of the expected values before diverging $\operatorname{Pr}_{t} \rightarrow \pm \infty$. As opposed to the flat channel case, where $\operatorname{Pr}_{t}$ is unbounded about the channel centerline, 


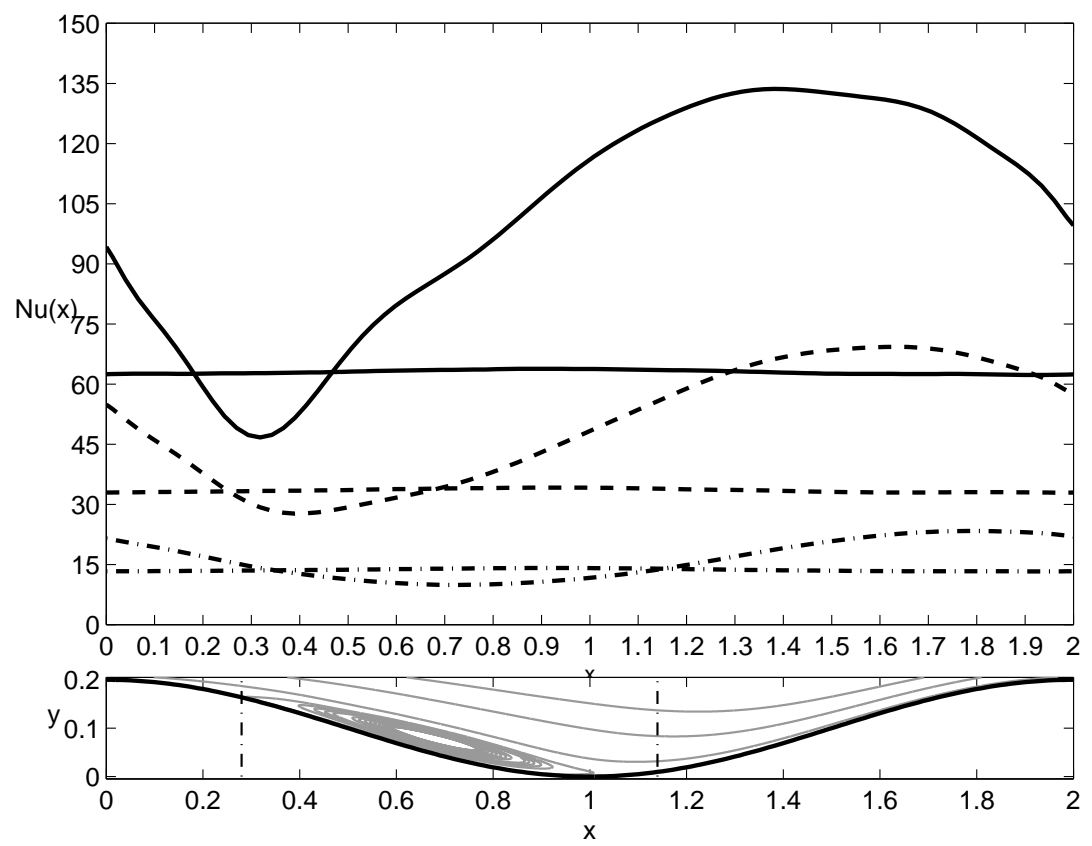

Figure 12: Profiles of the local Nusselt number: curves are for the lower wall while the almost horizontal lines are for the upper, flat wall. The solid line indicates $\mathrm{Nu}(x)$ for $\operatorname{Pr}=0.71$, the dashed line is for $\operatorname{Pr}=0.20$, the dash-dot line for $\operatorname{Pr}=0.025$.

in the present case unboundedness occurs also also close to the walls. The unbounded regions are of wider extension for $\operatorname{Pr}=0.025$, where the $\operatorname{Pr}_{t}$ distribution is also less uniform. Outside the unbounded regions, $\operatorname{Pr}_{t}$ is seen to be smaller than its space averaged value close to separation (see the region about $x=0.3$ for $y$ slightly above 0.2 ), this can be justified by Reynolds stress of comparatively small values and large turbulent heat fluxes. The observation is in agreement with the $\operatorname{Pr}_{t}$ behavior in separated flows found in the literature, see Ref. [34] and [35].

Space averages of the turbulent Prandtl number are given in table 4 using data of the present DNS; as there are regions in the field where the local $\operatorname{Pr}_{t} \rightarrow$ $\pm \infty$, these are evaluated omitting from the calculation all computational cells where $\operatorname{Pr}_{t}<0.50$ or $\operatorname{Pr}_{t}>3.3$.

The assumption of a uniform value of $\mathrm{Pr}_{t}$ is included in the hypothesis of most turbulence models for the closure of RANS equations. This is also the case of the Simple Gradient Diffusion Hypothesis (SGDH), which is probably the most widely used turbulent heat flux model. In view of the discussion 


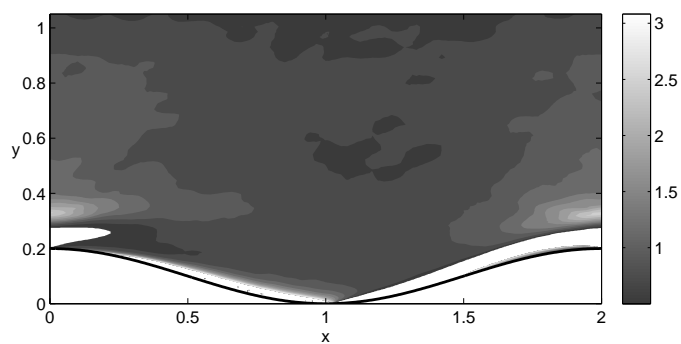

(a) $\operatorname{Pr}=0.71$

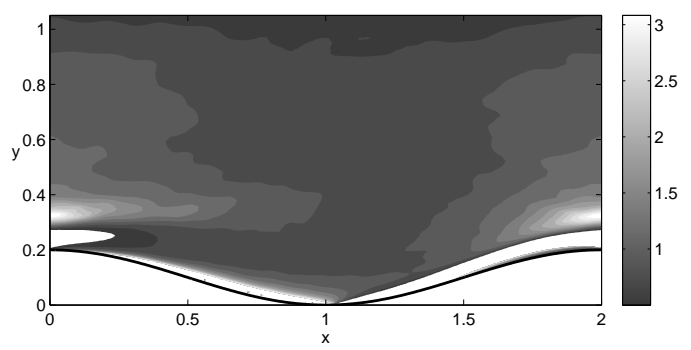

(b) $\operatorname{Pr}=0.20$

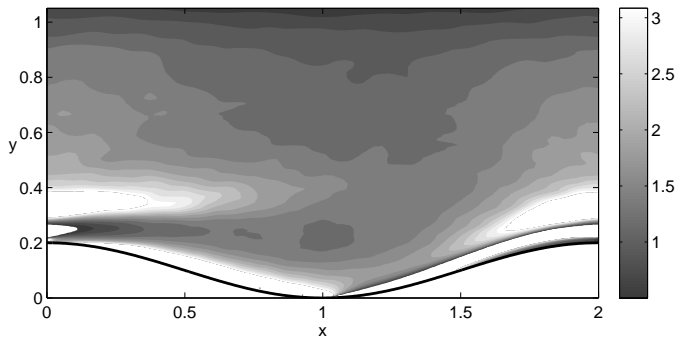

(c) $\operatorname{Pr}=0.025$

Figure 13: Contour plots of the time-averaged turbulent Prandtl number over the wavy walls. White regions indicate $\operatorname{Pr}_{t}<0.50$ or $\operatorname{Pr}_{t}>3.3$. Values of $\left\langle\operatorname{Pr}_{t}\right\rangle$ for the three molecular Pr are given in table 4.

above, where it is shown that flow separation gives place to a lower $\operatorname{Pr}_{t}$ region close to the separation point and introduces regions where $\operatorname{Pr}_{t}$ is unbounded close to the wall, an in-depth analysis of the SGDH model performances in the present case appears to be of interest. This is the topic of the following section, where in addition the effect of molecular Prandtl number below one is considered. 


\subsubsection{On the Simple Gradient Diffusion Hypothesis}

If by one side the gradient diffusion hypothesis is often considered too simplistic also for simple flows (see Corrsin [36] and Kim and Moin [37]), on the other side there are studies that demonstrate that it can provide reasonable results, see for example Ref. [38]. Moreover more complicated approaches, like the high-order stress transport closure (see for example Ref. [39]) give more accurate results only for few, selected cases. This can help explain the wide use of simple gradient models in the industrial practice.

In this paragraph, results obtained from the DNS are used as reference data for assessing the accuracy of turbulent heat fluxes predicted by the SGDH model in the present geometry and conditions, thus performing an a priori analysis of the model. The SGDH model is based on the gradient diffusion hypothesis

$$
\overline{u_{i}^{\prime} T^{\prime}}=-\frac{\nu_{t}}{\widehat{\operatorname{Pr}}_{t}} \frac{\partial \bar{T}}{\partial x_{i}}
$$

where $\widehat{\operatorname{Pr}}_{t}$ indicates an assigned value for the turbulent Prandtl number. In order to single out errors associated with turbulent transport modeling, DNS data are used in equation (17) for both temperature gradient and eddy viscosity to form the modeled heat fluxes.

Figure 14 displays turbulent heat flux vectors from DNS and from the SGDH model using $\widehat{\operatorname{Pr}}_{t}=0.9$. Given the isotropic character of the gradient diffusion hypothesis, the model fails to represent the streamwise component of turbulent heat fluxes. By comparing differences between the vertical component of heat fluxes it is instead noticed that the SGDH provides more accurate predictions of $\overline{v^{\prime} T^{\prime}}$ at $\operatorname{Pr}=0.71$, with respect to the $\operatorname{Pr}=0.20$ and $\operatorname{Pr}=0.025$ cases. This is to be ascribed to the fact that the usually suggested value of 0.9 for parameter $\widehat{\operatorname{Pr}}_{t}$ is close to the average $\operatorname{Pr}_{t}$ value for fluids of molecular Pr number around unity. For more accurate predictions, the assigned $\widehat{\operatorname{Pr}}_{t}$ value should be selected taking into account the molecular Prandtl number and possibly also flow regime.

The model by Piller et al. [3] provides the ratio between turbulent diffusivity values at different friction Péclet numbers and writes

$$
\frac{\left\langle\alpha_{t}\right\rangle}{\left\langle\alpha_{t}\right\rangle_{\operatorname{Pr}=1}}=1-\exp \left(-C \operatorname{Pe}_{\tau}^{n}\right)
$$

where $C=0.232$ and $n=0.574$. Being dependent on both flow regime and fluid characteristics, equation (18) is a good candidate for providing $\widehat{\operatorname{Pr}}_{t}$ 


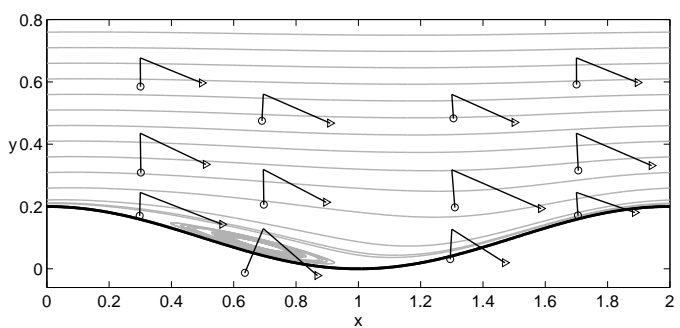

(a) $\operatorname{Pr}=0.71$

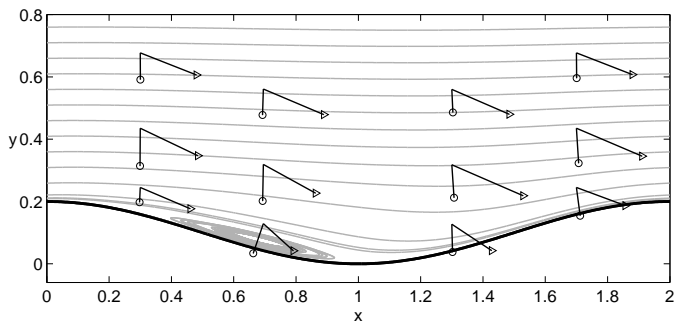

(b) $\operatorname{Pr}=0.20$

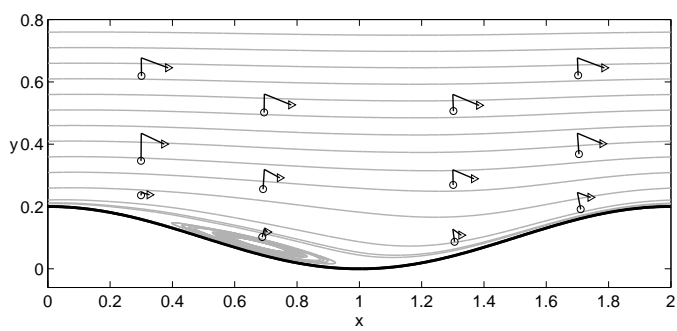

(c) $\operatorname{Pr}=0.025$

Figure 14: Comparison between vectors of turbulent heat fluxes, for the three different Pr. Triangle head: DNS results; round head: SGDH model using $\widehat{\mathrm{Pr}}_{t}=0.9$ for all Pr.

to be employed in models. In the present work, the model by Piller and coworkers [3] is used under the hypothesis that $\left\langle\operatorname{Pr}_{t}\right\rangle$ equals 0.9 for $\operatorname{Pr}=0.71$ giving place to the following equation

$$
\widehat{\operatorname{Pr}}_{t}=0.9 \frac{1-\exp \left(-C \mathrm{Pe}_{\tau, 0.71}^{n}\right)}{1-\exp \left(-C \mathrm{Pe}_{\tau}^{n}\right)}
$$

values calculated are compared to simulation results in table 4.

Figure 15 shows the comparison of $\overline{v^{\prime} T^{\prime}}$ profiles as calculated by DNS against the SGDH model results using both $\widehat{\operatorname{Pr}}_{t}=0.9$ and the $\widehat{\operatorname{Pr}}_{t}$ formulation 


\begin{tabular}{lcc} 
Pr & $\begin{array}{c}\left\langle\operatorname{Pr}_{t}\right\rangle \\
\text { present DNS }\end{array}$ & $\begin{array}{c}\widehat{\operatorname{Pr}}_{t} \\
\text { Eq. }(19)\end{array}$ \\
\hline 0.71 & 0.861 & 0.9 \\
0.20 & 0.924 & 0.987 \\
0.025 & 1.22 & 1.754 \\
\hline
\end{tabular}

Table 4: Turbulent Prandtl number values at $\operatorname{Re}_{\tau}=282$.

of equation (19). For decreasing Prandtl number the SGDH model used in conjunction with equation (19) is seen to better approximate DNS results, showing a good improvement with respect to the assigned $\widehat{\mathrm{Pr}}_{t}=0.9$ approach especially for $\operatorname{Pr}=0.025$. Furthermore, for decreasing Prandtl number, discrepancies between DNS and models are seen to move from the lower wall towards the channel center. This is due to the fact that the predicted heat fluxes and also errors are largest where the gradient of the mean field is largest, see also the discussion by Grotzbach [40].

It is also observed in figure 15 that for $x=0.7$, turbulent heat fluxes predicted by equation (17) are very unlike heat fluxes calculated by DNS, especially close to the lower wall. The abrupt change of sign of fluxes predicted is due to the change of sign of $\partial \bar{u} / \partial y$ taking place within the recirculating region which is reflected in the $\nu_{t}$ behavior in equation (17). A similar discrepancy in the sign of vertical turbulent heat fluxes is observed also about $x=1.7$, this instead has to be attributed to the change of sign of Reynolds stress calculated in the global reference frame, see figure 5. In both cases discussed, the discrepancy is due to an exception in the sign of $\nu_{t}$ in equation (17) calculated from DNS as the ratio between Reynolds stress and $y$-derivative of $\bar{u}$. None of the two exceptions should occur when a SGDH model is used in conjunction with a modeled $\nu_{t}$ field, which is usually calculated as positive. This does not mean that errors expected on the Reynolds averaged temperature field in those regions are small; instead errors are expected on the temperature field which will derive from an inaccurate calculation of the Reynolds averaged velocity field.

\section{Conclusions}

Forced convective heat transfer in turbulent regime is simulated between a flat wall and a wavy wall; three are the fluids considered, of Prandtl numbers $\operatorname{Pr}=0.71,0.20,0.025$. The simulated friction Reynolds number is in all cases 

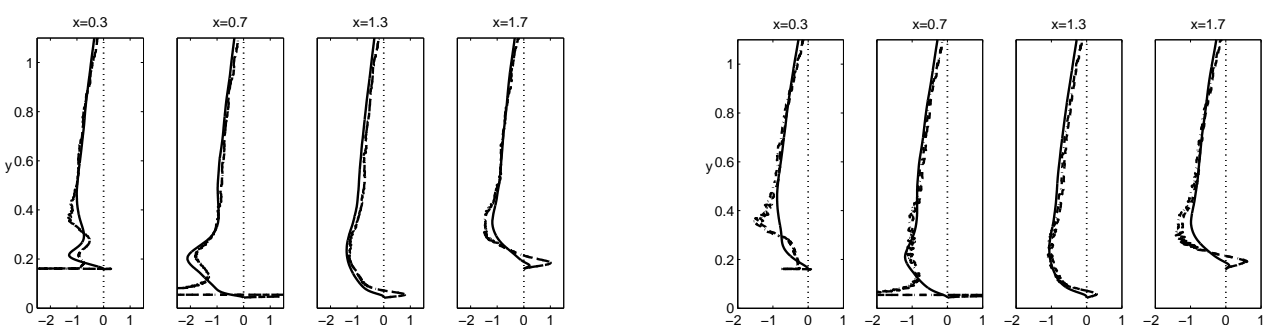

(a) $\operatorname{Pr}=0.71$

(b) $\operatorname{Pr}=0.20$
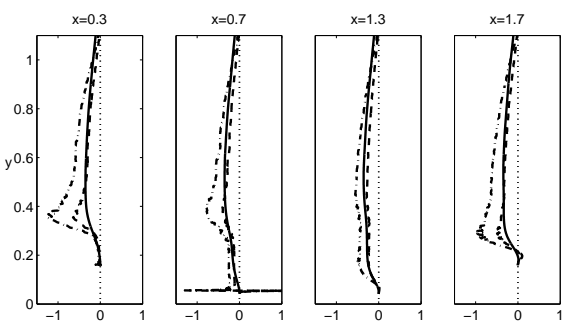

(c) $\operatorname{Pr}=0.025$

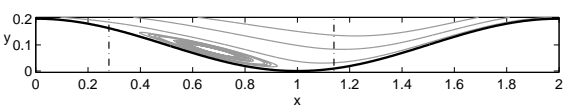

Figure 15: Comparison between turbulent heat fluxes in $y$ direction, for the three different Pr. Solid line: results by DNS; dashed line: results by SGDH model with ${\widehat{\mathrm{Pr}_{t}}}_{t}$ as in equation (19); dash-dot line: results by SGDH model using $\widehat{\mathrm{Pr}}_{t}=0.9$ for all $\mathrm{Pr}$.

$\operatorname{Re}_{\tau}=282$, corresponding to a Reynolds number of the bulk velocity and the hydraulic diameter, $R e=18875$. In this flow regime the lower, undulated wall induces separation. A shear layer, which generates from the crest of the wave, is located above the mean recirculation region. For $\operatorname{Pr}=0.71$ and $\operatorname{Pr}=0.20$ an inflection of the mean temperature profile is observed just above the separation bubble. This characteristic feature, which is mainly due to advective transport, is not observed for $\operatorname{Pr}=0.025$.

For $\operatorname{Pr}=0.71$, the Nusselt number is minimum within the separated flow region, while it reaches its maximum in the up-slope part of the wave. The same behavior is observed also for low Prandtl number fluids, but with smaller deviations from the space-averaged Nusselt number. The heat transfer effectiveness of low Prandtl number fluids is lower with respect to the $\operatorname{Pr}=0.71$ case but the heat transfer rate is in all cases enhanced by the wall undulation.

The turbulent Prandtl number distribution is observed to become less uniform for decreasing Prandtl numbers, moreover separation and reattachment determine an unbounded behavior of $\mathrm{Pr}_{t}$ in wide regions close to the 
channel walls. These characteristics represent potential sources of errors when turbulence models are employed, which involve the specification of a given, uniform $\widehat{\operatorname{Pr}}_{t}$ value.

Predictive performances of the SGDH model for the evaluation of vertical heat fluxes are assessed in the conditions of the present study. Results suggest that vertical heat fluxes can be well represented also in low-Prandtl number fluids provided this is taken into account by selecting the $\widehat{\operatorname{Pr}}_{t}$ value according to the molecular Pr. The model by Piller and coworkers [3] which has been used for determining $\widehat{\operatorname{Pr}}_{t}$ provides the ratio between turbulent diffusivities at different friction Péclet numbers and therefore also flow regime can be accounted for. In the separation region as well as downstream of flow reattachment the a priori analysis performed on the SGDH model calculates wall normal heat fluxes diverging from the correct behavior. While such discrepancies are not expected to be reproduced in practical applications where models for eddy viscosity do not consider negative values, errors are expected on the temperature field which will be inherited from an inaccurate calculation of the Reynolds averaged velocity field.

\section{Acknowledgements}

This research is funded by THINS, a European funded large-scale collaborative project within the VII Framework Program (project number 249337).

\section{A. Validation against experiments and previous simulations}

In this section present results are compared to experimental data from Ref. [6] and DNS data from Ref. [10]. Reference data have been taken directly from figures 10a and 10b in Ref. [10].

The Reynolds number based on the mean velocity and the hydraulic diameter of the simulation used for validation purposes $R e=13732$, does not match exactly the Reynolds number of reference data, $\mathrm{Re}=13840$ because in the numerical methodology implemented for the present study, the forcing term is the given parameter while the average velocity is a result of the simulations.

Figure 16 displays all comparisons performed. Profiles of the main dynamical quantities are seen to be well represented by the present simulations, when compared to both experiments and numerical simulations. Flow separation and reattachment locations are compared to Ref. [10] in Table 5. 


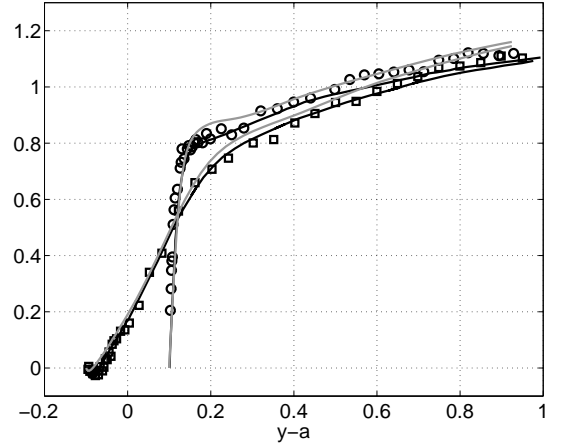

(a) $\frac{u}{u_{m}}$

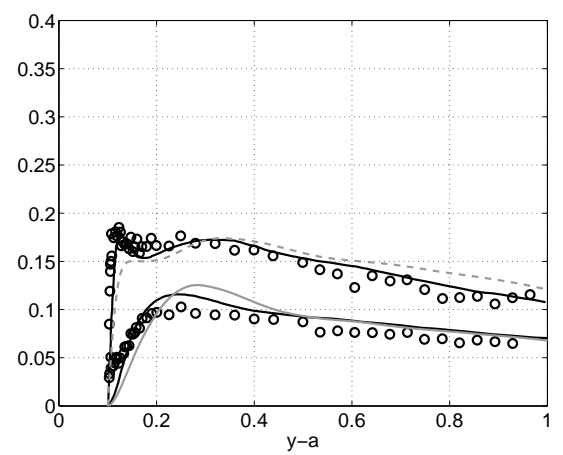

(c) $\frac{u_{\mathrm{rms}}}{u_{m}}, \frac{v_{\mathrm{rms}}}{u_{m}}$

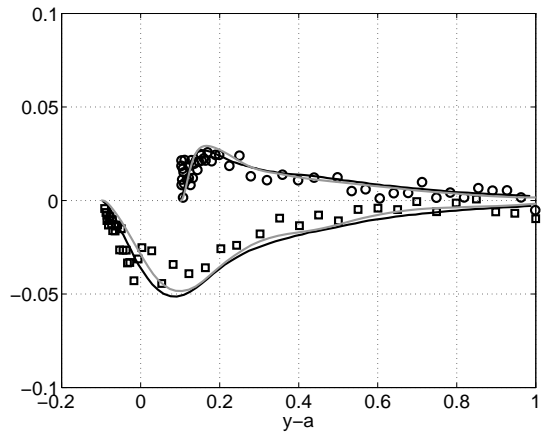

(b) $\frac{v}{u_{m}}$

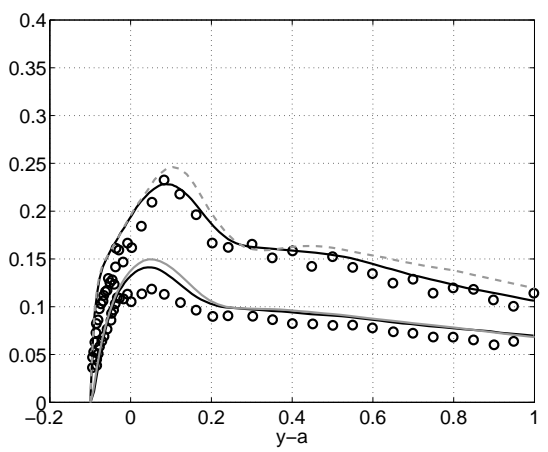

(d) $\frac{u_{\mathrm{rms}}}{u_{m}}, \frac{v_{\mathrm{rms}}}{u_{m}}$

Figure 16: Validation of the numerical technique employed by comparison with experiments and DNS results from the literature. (a) Profiles of the normalized mean streamwise velocity for crest and trough. Gray lines indicate present results; symbols indicate measurements in Ref. [6]; solid black lines are for the DNS in Ref. [10]. (b) Profiles of the normalized mean vertical velocity for crest and trough. Legend is like for figure 16a. (c) Profiles of the normalized root-mean-square velocity fluctuations at the crest. The dashed gray line indicates $u_{\mathrm{rms}} / u_{m}$, the solid gray line is for $v_{\mathrm{rms}} / u_{m}$; symbols indicate measurements in Ref. [6]; dashed lines are for the DNS in Ref. [10]. (d) Profiles of the normalized root-mean-square velocity fluctuations at the trough; legend is like for figure 16c

\section{References}

[1] H. Kawamura, K. Ohsaka, H. Abe, K. Yamamoto, DNS of turbulent heat transfer in channel flow with low to medium-high Prandtl number fluid, Int. J. Heat Fluid Flow 19 (1998) 482-491. 


\begin{tabular}{lcc} 
& $x_{s} / \lambda$ & $x_{r} / \lambda$ \\
\hline Present & 0.14 & 0.57 \\
{$[10]$} & 0.14 & 0.59 \\
\hline
\end{tabular}

Table 5: Separation and reattachment positions of the mean flow, comparison between DNS results.

[2] H. Kawamura, H. Abe, Y. Matsuo, DNS of turbulent heat transfer in channel flow with respect to Reynolds and Prandtl number effects, International Journal of Heat and Fluid Flow 20 (3) (1999) 196-207.

[3] M. Piller, E. Nobile, T. Hanratty, DNS study of turbulent transport at low Prandtl numbers in a channel flow, J. Fluid Mech. 458 (2002) 419-441.

[4] H. Kawamura, H. Abe, Y. Matsuo, Surface heat-flux fluctuation in a turbulent channel flow up to $\operatorname{Re}_{\tau}=1020$ with $\operatorname{Pr}=0.025$ and 0.71, Int. J. Heat Fluid Flow 25 (2004) 404-419.

[5] D. Zilker, T. Hanratty, Influence of the amplitude of a solid wavy wall on a turbulent flow. Part 2. separated flows, J. Fluid Mech. 90 (1979) $257-271$.

[6] J. D. Hudson, The effect of a wavy boundary on turbulent flow, Ph.D. thesis, University of Illinois (1993).

[7] J. D. Hudson, L. Dykhno, T. J. Hanratty, Turbulence production in flow over a wavy wall, Experiments in Fluids 20 (1996) 257-265.

[8] S. Kuhn, C. Wagner, P. R. von Rohr, The influence of wavy walls on the transport of a passive scalar in turbulent flows, Journal of Turbulence 9 (10) (2008) 1-17.

[9] C. Maaß, U. Schumann, Direct numerical simulation of separated turbulent flow over a wavy boundary, in: E. H. Hirschel (Ed.), Flow simulation with high-performance computers, Vieweg, DLR, Institut fur Physik der Atmosphare D-82230 Oberpfanffenhofen, Germany, 1996.

[10] P. Cherukat, Y. Na, T. J. Hanratty, Direct numerical simulation of a fully developed turbulent flow over a wavy wall, Theoret. Comput. Fluid Dynamics 11 (1998) 109-134. 
[11] R. J. Calhoun, R. L. Street, Turbulent flow over a wavy surface: Neutral case, J. Geophys. Res. 106 (2001) 9277-9293.

[12] V. Patel, J. Cohn, J. Yoon, Turbulent flow in a channel with a wavy wall, Journal of Fluids Engineeering 113 (1991) 579-586.

[13] N. Kruse, S. Kuhn, P. R. von Rohr, Wavy wall effects on turbulence production and large-scale modes, Journal of Turbulence 7 (2006) 1-28.

[14] S. Kuhn, C. Wagner, P. R. von Rohr, Influence of wavy surfaces on coherent structures in a turbulent flow, Experiments in Fluids 43 (2007) 251-259.

[15] A.Günther, P. R. von Rohr, Structure of the temperature field in a flow over a heated waves, Experiments in Fluids 33 (2002) 920-930.

[16] H. S. Choi, K. Suzuki, Large eddy simulation of turbulent flow and heat transfer in a channel with one wavy wall, Int. J. Heat Fluid Flow 26 (2005) 681-694.

[17] C. Wagner, S. Kenjereš, P. R. von Rohr, Dynamic large eddy simulations of momentum and wall heat transfer in forced convection over wavy surfaces, Journal of Turbulence 12 (2010) 1-27.

[18] R. Rossi, Passive scalar transport in turbulent flows over a wavy walls, Ph.D. thesis, Università degli studi di Bologna (2006).

[19] A. Z. Dellil, A. Azzi, B. A. Jubran, Turbulent flow and convective heat transfer in a wavy wall channel, Heat and Mass Transfer 40 (2004) 793799 .

[20] B. J. Daly, F. H. Harlow, Transport equations in turbulence, Phys. Fluids 13 (1970) 2634-2649.

[21] K. Abe, K. Suga, Towards the development of a Reynolds-averaged algebraic turbulent scalar-flux model, Int. J. Heat Fluid Flow 22 (2001) $19-29$.

[22] R. Rossi, A numerical study of algebraic flux models for heat and mass traansport simulation in complex flows, Int. J. Heat Mass Transfer 53 (2010) 4511-4524. 
[23] W. Kays, Turbulent Prandtl number-where are we?, J. Heat Transfer 116 (1994) 284-295.

[24] D. McEligot, M. Taylor, The turbulent Prandtl number in the near-wall region for low-Prandtl-number gas mixtures, Int. J. Heat Mass Transfer 39 (1996) 1287-1295.

[25] X. Cheng, N. Tak, Investigation on turbulent heat transfer to leadbismuth eutectic flows in circular tubes for nuclear applications, Nucl. Eng. Des. 236 (2006) 385-393.

[26] F. Chen, X. Huai, J. Cai, X. Li, R. Meng, Investigation on the applicability of turbulent-Prandtl-number models for liquid lead-bismuth eutectic, Nucl. Eng. Des. 257 (2013) 128-133.

[27] E. Stalio, M. Piller, Direct numerical simulation of heat transfer in converging-diverging wavy channels, ASME Journal of Heat Transfer 129 (7) (2007) 769-777.

[28] E. Stalio, E. Nobile, Direct numerical simulation of heat transfer over riblets, Int. J. Heat Fluid Flow 24 (3) (2003) 356-371.

[29] E. Stalio, D. Angeli, G.S. Barozzi, Numerical simulation of forced convection over a periodic series of rectangular cavities at low Prandtl number, Int. J. Heat Fluid Flow 32 (5) (2011) 1014-1023.

[30] A. Günther, P. R. von Rohr, Large-scale structures in a developed flow over a wavy wall, J. Fluid Mech. 478 (2003) 257-285.

[31] R. Moser, J. Kim, N. Mansour, Direct numerical simulation of turbulent channel flow up to $\operatorname{Re}_{\tau}=590$, Phys. Fluids 11 (4) (1999) 943-945.

[32] M. S. Bhatti, R. K. Shah, Turbulent and transition flow convective heat transfer in ducts, in: S. Kakac, R. K. Shah, W. Aung (Eds.), Handbook of Single-Phase Convective Heat Transfer, John Wiley and Sons, 1987, Ch. 4.

[33] C. A. Sleicher, M. W. Rouse, A convenient correlation for heat transfer to constant and variable property fluids in turbulent pipe flow, Int. J. Heat Mass Transfer 18 (1975) 667-683. 
[34] Y. Nagano, H. Hattori, T. Houra, DNS of velocity and thermal fields in turbulent channel flow with transverse-rib roughness, Int. J. Heat Fluid Flow 25 (2004) 393-403.

[35] R. Avancha, R. H. Pletcher, Large eddy simulation of the turbulent flow past a backward-facing step with heat transfer and property variations, Int. J. Heat Fluid Flow 23 (2002) 601-614.

[36] S. Corrsin, Limitations of gradient transport models in random walks and in turbulence, Adv.Geophys 18/A (1974) 25-60.

[37] J. Kim, P. Moin, Transport of passive scalars in a turbulent channel flow, in: F.Durst (Ed.), Turbulent Shear Flows 6, Springer-Verlag, New York, 1989, pp. 85-96.

[38] M. Draksler, B. Koncar, Analysis of heat transfer and flow characteristics in turbulent impinging jet, Nucl. Eng. Des. 241 (2011) 1248-1254.

[39] C. Wang, Y. Jang, M. A. Leschziner, Modelling two- and threedimensional separation from curved surfaces with anisotropy resolving turbulence closures, Int. J. Heat Fluid Flow 25 (2004) 499-512.

[40] G. Grötzbach, Challenges in low-Prandtl number heat transfer simulation and modelling, Nucl. Eng. Des. 264 (2013) 41-55. 